\title{
Infants' perception of rhythm and tempo in unimodal and multimodal stimulation: A developmental test of the intersensory redundancy hypothesis
}

\author{
LORRAINE E. BAHRICK and ROBERT LICKLITER \\ Florida International University, Miami, Florida
}

\begin{abstract}
Research has demonstrated that young infants can detect a change in the tempo and the rhythm of an event when they experience the event bimodally (audiovisually), but not when they experience it unimodally (acoustically or visually). According to Bahrick and Lickliter (2000, 2002), intersensory redundancy available in bimodal, but not in unimodal, stimulation directs attention to the amodal properties of events in early development. Later in development, as infants become more experienced perceivers, attention becomes more flexible and can be directed toward amodal properties in unimodal and bimodal stimulation. The present study tested this developmental hypothesis by assessing the ability of older, more perceptually experienced infants to discriminate the tempo or rhythm of an event, using procedures identical to those in prior studies. The results indicated that older infants can detect a change in the rhythm and the tempo of an event following both bimodal (audiovisual) and unimodal (visual) stimulation. These results provide further support for the intersensory redundancy hypothesis and are consistent with a pattern of increasing specificity in perceptual development.
\end{abstract}

Infants encounter a world of dynamic multimodal stimulation to all the senses. People and objects provide an array of changing, concurrent, tactile, visual, auditory, gustatory, and olfactory stimulation. For example, a person provides synchronous auditory and visual information during speech. In contrast, other events provide unimodal stimulation and reach only a single sense. A person speaking from a nearby room can be heard but not seen. What do infants selectively attend, perceive, and learn from the constantly changing, richly structured flux of stimulation to the various senses? How does this change developmentally so that infants rapidly develop the intermodal knowledge, categories, and expectations of adult perceivers?

According to Gibson (1969), infants perceive the world through a unified perceptual system, and they detect amodal information that unites stimulation across the senses. Amodal information is information, such as synchrony, rhythm, tempo, and intensity, that can be detected in more than one sense modality. Detecting this information promotes the processing of unitary multimodal events in young infants. Through development, infants differentiate increasingly finer aspects of stimulation (Gibson,

This research was supported by NIMH Grants RO1 MH62226 and RO1 MH62225, awarded to the first and second authors, respectively. We gratefully acknowledge Katryna Anasagasti, Laura Batista, Irina Castellanos, Ross Flom, Melissa Shuman, and Mariana Vaillant for their assistance with data collection and analyses. Address all correspondence to L.E. Bahrick, Department of Psychology, Florida International University, Miami, FL 33199 (e-mail: bahrick@fiu.edu).
1969). During the past 3 decades, a growing body of research has supported this view and has demonstrated that infants are capable of perceiving unitary multimodal events by detecting a host of amodal properties of stimulation. For example, young infants detect amodal information uniting faces and voices, including voice-lip synchrony, rhythm, tempo, and spectral information common to the movements of the lips and the sounds of speech (e.g., Dodd, 1979; Kuhl \& Meltzoff, 1984; Lewkowicz, 1996, 2000), as well as audible and visible information specifying emotion (see Walker-Andrews, 1997, for a review). Infants are also able to match objects and sounds on the basis of temporal synchrony, tempo, rhythm, and temporal microstructure specifying the substance and composition of objects (e.g., Bahrick, 1983, 1987, 1988; Lewkowicz, 2000; Spelke, 1979; Spelke, Born, \& Chu, 1983). This ability to detect the amodal properties of multimodal events promotes the perception of coherent unitary events from stimulation to different senses in early development.

There is also a growing body of neurophysiological and psychophysical evidence for the salience of amodal information (Calvert, Spence, \& Stein, 2004; Shimojo \& Shams, 2001). For example, spatially colocated auditory and visual stimulation elicits neural responses that are greater than the sum of the neural responses to the unimodal components considered separately (reviewed in Stein \& Meredith, 1993). These types of findings have led to a growing appreciation of the brain's sensitivity to amodal information (see Calvert et al., 2004, for examples) and have provided important insights into the neural substrates underlying the detection of multimodal information 
during infancy and beyond (see Lickliter \& Bahrick, 2000).

Not all information, however, is amodal. Objects and events make both amodal and modality-specific information available. A speaking person provides amodal rhythm, tempo, and synchrony, common to the sight of the face and the sound of the voice, as well as information specific to one sense, such as the pattern and configuration of the face or the pitch and timbre of the voice. Infants also rapidly become skilled perceivers of this modality-specific information. For example, they discriminate between the faces of different individuals (see, e.g., Bahrick, Moss, \& Fadil, 1996; Barrera \& Maurer, 1981; Caron, Caron, Caldwell, \& Weiss, 1973; Cohen \& Strauss, 1979; Fagan, 1972, 1976), as well as among different voices (Bahrick, Lickliter, Shuman, Batista, \& Grandez, 2003; DeCasper \& Fifer, 1980; Miller, 1983; Miller, Younger, \& Morse, 1982) in the first months of life. How is attention guided to the amodal versus the modality-specific aspects of stimulation, and how does this change developmentally?

In accord with Gibson's (1969) differentiation view of perceptual development, research has demonstrated that intersensory perception develops in the order of increasing specificity and that detection of amodal relations guides and constrains perceptual learning about arbitrary modality-specific relations (e.g., Bahrick, 1992, 1994, 2001, 2004). For example, 7-month-old infants detect the arbitrary relation between an object and a speech sound if the object is moved in synchrony with the sound, but not if it is still or out of synchrony (Gogate \& Bahrick, 1998). Other studies have also shown that infants learned intermodal relations between modality-specific properties of events in the presence, but not in the absence, of amodal relations (e.g., Bahrick, 1988; Hernandez-Reif \& Bahrick, 2001; Slater, Quinn, Brown, \& Hayes, 1999). Detection of amodal relations, such as temporal synchrony, leads to further exploration of the nature of the sights and sounds. This learning in the order of increasing specificity is adaptive because it can promote learning about regularities across the senses that are context independent prior to learning about specific relations that are context dependent (see Bahrick, 2001). An early processing precedence for amodal information can thus organize perceptual development and have far-reaching cascading effects on later attention, perception, learning, and memory (see Bahrick, 2004; Bahrick \& Lickliter, 2002; Bahrick, Lickliter, \& Flom, 2004a).

Recently, Bahrick and Lickliter $(2000,2002)$ provided evidence for an intersensory redundancy hypothesis (IRH) to describe the conditions under which infants selectively attend to amodal versus modality-specific properties of stimulation and how this influences early perceptual development. Intersensory redundancy is highly salient to young infants and directs attentional selectivity. Intersensory redundancy refers to the concurrent, spatially colocated presentation of the same information across two or more sensory systems (e.g., rate, rhythm, duration or intensity). For auditory-visual stimulation, it also entails temporal synchrony between the two sources of stimulation. According to the IRH, in early development, information presented redundantly to two or more senses simultaneously selectively recruits infants' attention and promotes perceptual learning of amodal stimulus properties more effectively than does the same amodal information presented to one sense modality alone. Thus, a rhythm can be perceived more easily if it is conveyed through two sense modalities rather than through one (Bahrick \& Lickliter, 2000). In contrast, when information is presented to one sense modality (e.g., visually), it selectively recruits attention to modality-specific properties more effectively than when the modality-specific properties are presented in the context of more than one sense modality (Bahrick, Lickliter, \& Flom, 2004b; Bahrick et al., 2003). Since most natural events are multimodal, this attentional selectivity gives an early developmental advantage to perceiving and learning on the basis of amodal properties prior to learning on the basis of modality-specific properties, consistent with a pattern of increasing specificity. This selective advantage for amodal information should be especially evident when the properties to be perceived are difficult for the infant to discriminate and/or are relatively novel.

A number of recent studies with both human and animal infants have explored this hypothesis and have provided support for the principle that amodal properties, such as rhythm and tempo, are at first more easily perceived when they are presented bimodally (i.e., audiovisually) than when they are presented unimodally (visually or acoustically; see Bahrick, Flom, \& Lickliter, 2002; Bahrick \& Lickliter, 2000; Bahrick et al., 2004a; Lickliter, Bahrick, \& Honeycutt, 2002, 2004). For example, Bahrick and Lickliter (2000) habituated 5-month-old infants to videos of a hammer tapping out a complex rhythm under bimodal (synchronized vs. nonsynchronized audiovisual) or unimodal (acoustic or visual) conditions. The results indicated that only the infants who received the synchronized bimodal presentation discriminated the novel from the familiar rhythm, whereas those who received unimodal visual, unimodal auditory, or bimodal nonsynchronized presentations did not. Bahrick et al. (2002) extended this test to the amodal property of tempo and to infants of a younger age. Three-month-old infants were habituated to videos of the hammer tapping a rhythm in one of two tempos (slow vs. fast) under conditions of bimodal stimulation (synchronized audiovisual) versus unimodal stimulation (acoustic or visual). The results replicated and extended those of Bahrick and Lickliter (2000), demonstrating that the infants could discriminate a change in tempo following synchronous bimodal, but not unimodal, exposure. These findings support the primary prediction of the IRH and demonstrate that only infants who received intersensory redundancy showed evidence of discriminating the amodal properties of rhythm and tempo.

Comparative research has also demonstrated that animal embryos and infants show a dramatic advantage in perceptual learning when they receive redundant bimodal, as contrasted with unimodal, stimulation. Lickliter, Bahrick, 
and Honeycutt $(2002,2004)$ tested bobwhite quail chicks for their preference between one of two variants of a species-typical maternal call. Chicks that received prenatal redundant audiovisual exposure (a patterned light that shared synchrony, rhythm, and tempo with the sounds of the call) as embryos learned the call four times faster and remembered it four times longer than those that received unimodal auditory exposure. Furthermore, asynchronous bimodal presentations during the prenatal period interfered with perceptual learning and memory. These findings converge with the results from studies of human infants and suggest that the selective advantage of intersensory redundancy for detecting amodal properties of stimulation is likely a general developmental principle that serves to organize and constrain early perceptual development across species and across the prenatal and the early postnatal periods.

To what extent do the effects of intersensory redundancy persist across later stages of development? According to the IRH, the advantage of intersensory redundancy for the perceiving of amodal properties should be most pronounced during early development and when stimulation is relatively new and/or tasks are difficult. With experience, as infants become more skilled perceivers, attention becomes more flexible, and perception of amodal properties (such as rhythm or tempo) should no longer require intersensory redundancy (unless the task is difficult). Amodal properties may then be detected in unimodal, as well as multimodal, stimulation. This hypothesized developmental progression from early detection of amodal properties across two or more senses (intermodal) to later detection of amodal properties through one sense alone (intramodal) is another example of progressive differentiation in the order of increasing specificity in perceptual development.

In the present study, we explored this developmental progression. We replicated and extended our two prior studies (Bahrick et al., 2002; Bahrick \& Lickliter, 2000), in which infants of 3 and 5 months demonstrated detection of tempo and rhythm, respectively, following redundant bimodal stimulation, but not following unimodal stimulation. We asked whether older infants would detect these amodal properties in both bimodal (audiovisual) and unimodal visual stimulation, given their additional months of experience with natural events in the world. It was hypothesized that if perception of amodal properties emerges in the context of redundancy and is later extended to nonredundant unimodal contexts, older, more experienced infants should no longer require intersensory redundancy for detecting rhythm and tempo changes.

\section{EXPERIMENT 1 \\ Discrimination of Tempo in Redundant Bimodal Stimulation Versus Unimodal Visual Stimulation}

In this experiment, we investigated the ability of 5month-old infants to perceive a change in the amodal property of tempo in redundant bimodal (auditory and vi- sual) stimulation versus nonredundant unimodal visual stimulation, using procedures and stimuli similar to those in Bahrick et al., (2002). To assess unimodal discrimination of tempo, we focused on unimodal visual discrimination (rather than unimodal auditory discrimination) for practical reasons. In our prior studies, the young infants were equally poor at discriminating unimodal visual and unimodal auditory changes in tempo (Bahrick et al., 2002) and rhythm (Bahrick \& Lickliter, 2000). Furthermore, since our dependent variable was visual fixation time, assessing visual discrimination allowed for a more direct measure of discrimination than did assessing auditory discrimination. Thus, the infants in the present experiment received bimodal audiovisual or unimodal visual habituation to a videotaped event consisting of a hammer tapping out a rhythm at one of two tempos. Following habituation, the infants received test trials in which a change in tempo under their respective conditions was depicted, and we assessed their visual recovery to the change.

\section{Method}

Participants. Thirty-two 5-month-old infants participated. There were 18 females and 14 males, and they had a mean age of 157.4 days $(S D=6.4)$. The infants were primarily from middle class homes, and their parents had at least a high school education. All the infants had a gestation period of at least 38 weeks. Seven were Caucasian, 23 were of Hispanic origin, and 2 were African American. Seven additional infants participated $(18 \%)$, but their data were excluded from the analyses due to fussiness $(n=1)$, failure to habituate within 20 trials $(n=1)$, and failure to meet the fatigue $(n=3)$, and habituation $(n=2)$ criteria (see the Procedures section for details).

Stimulus Events. The stimulus events were taken from videotapes used in two prior studies (Bahrick et al., 2002; Bahrick \& Lickliter, 2000). They depicted a bright red toy hammer moving up and down, tapping out one of two rhythms at one of two tempos. The rhythms had an irregular temporal structure and differed in terms of their arrangement of elements. Each rhythm was composed of one whole-beat rest and four impacts ( 2 whole beats and 2 half beats) and each 4-beat rhythm alternated with a 4-beat measure of rests. The rhythms were $\mathrm{x} x \mathrm{o} \mathrm{xx}$ and $\mathrm{x}$ o xx x (where $\mathrm{x}$ represents a whole-beat impact, $x x$ two half-beat impacts, and o a whole-beat rest). Each rhythm was presented at one of two tempos (not taking into account the 4-beat measures of rests), 110 beats per minute $(1.8 \mathrm{~Hz})$ or 240 beats per minute $(4 \mathrm{~Hz})$. A control display depicted a green-and-white toy turtle whose arms spun, making a whirring sound.

Apparatus and Procedure. The apparatus and procedure were the same as those used in Bahrick, et al. (2002). The infants sat in a standard infant seat facing a 19-in. (Panasonic BT-S1900N) video monitor $55 \mathrm{~cm}$ away. The seat and monitor were surrounded by black curtains and black posterboard containing two apertures that allowed two observers to monitor the infants' visual fixations. The events were presented using a Panasonic (VHS NV-A500) edit controller connected to three Panasonic video decks (AG 6300 and AG 7750). The three video decks and edit controller enabled us to switch between the habituation, test, and control events without extraneous noise and time that would have resulted from changing cassettes. Soundtracks were presented from a speaker located just beneath the video monitor at approximately $65 \mathrm{~dB}$ (A scale, fast response), as measured from the infant seat.

A trained observer, unaware of the infant's condition and unable to see the video display, monitored the infants' visual fixations by depressing and holding a button as long as the infant was visually fixating the display. The button box was connected to a computer 
that recorded and computed the duration of the infants' visual fixations to the video displays and signaled a second experimenter regarding when to commence and terminate each trial. A record of the infants' visual fixations was created on line. The observations of the primary observer controlled the audiovisual presentations, and those of the secondary observer were recorded for later calculation of interobserver reliability.

The infants were tested with an infant control habituation procedure (Horowitz, Paden, Bhana, \& Self, 1972) identical to the bimodal and unimodal visual conditions of Bahrick et al. (2002), to determine whether they could detect a change in tempo following bimodal audiovisual $(n=16)$ or unimodal visual $(n=16)$ exposure to the events. The infants were randomly assigned to either the bimodal or the unimodal exposure condition. In the bimodal condition, the infants received an audible and visible presentation of the hammer tapping one of two rhythms at one of the two tempos. Test trials depicted the hammer tapping the same rhythm at a new tempo. In the unimodal condition, the infants received a silent visual presentation of the hammer tapping one of two rhythms at one of the two tempos. Test trials depicted the hammer silently tapping the same rhythm at a new tempo. Rhythm and tempo were counterbalanced across infants, so that half the infants in each condition (bimodal vs. unimodal) received one of the rhythms and, within each of these groups, half the infants were assigned one of the tempos for habituation and the other tempo for test.

The habituation procedure consisted of an initial control trial and 4 mandatory habituation trials and was terminated after the infant reached the habituation criterion and had performed 2 subsequent (no-change) posthabituation trials. Two test trials depicting a novel stimulus were then presented, followed by a final control trial. The length of each trial was controlled by the infants' looking behavior. Each trial began when the infant fixated the visual display and was terminated once the infant had looked away for $1.5 \mathrm{sec}$. In addition, $60 \mathrm{sec}$ was set as the maximum trial length and 20 trials as the maximum number of trials. The habituation criterion was defined as a decrement of $50 \%$ or greater on 2 consecutive trials, relative to the infant's initial interest level (baseline, the average fixation time during the first 2 habituation trials). Once the infants had met this criterion, 2 no-change posthabituation trials were presented. They were identical to the habituation trials and served as a basis for calculating visual recovery during test trials. By using posthabituation trials, we reduced chance habituation and ensured a more conservative criterion for habituation. We also took into account spontaneous regression effects by assessing visual recovery in relation to the posthabituation looking (see Bertenthal, Haith, \& Campos, 1983, for a discussion of regression effects).

We examined each infant's data to determine whether two criteria had been met, one to identify fatigue and the other to ensure that the infants who had reached the habituation criterion had, in fact, habituated (see Bahrick et al., 2002; Bahrick \& Lickliter, 2000). To make certain that the infants were not overly fatigued and unable to show visual recovery, their visual fixation to the control event (toy turtle) on the final trial was compared with that on the initial trial. A visual fixation to the toy turtle on the final trial that was at least $35 \%$ of the initial fixation level to the turtle was set as a minimum criterion for inclusion. The data of 2 infants were rejected for failure to meet this criterion. The remaining infants showed substantial visual fixation on the final control trial $(M=152 \%, S D=169 \%$, of the initial control trial). The infants' visual fixation to the toy turtle on the final trial was also required to be greater than their fixation to the habituated toy hammer events $(100 \%$ of posthabituation fixation level), in order to ensure that the infants were capable of showing visual recovery to a completely different event. The data of 1 infant were rejected for failure to meet this criterion. The remaining infants showed a substantial visual recovery on the final control trial, relative to their posthabituation fixation $(M=938 \%, S D=$ $663 \%$ ). In addition, to evaluate whether the infants had, in fact, habituated to the events, we compared the infants' mean posthabitua- tion fixation levels with their mean initial fixation levels (baseline). The data of any infant whose posthabituation fixation exceeded the baseline fixation level were excluded $(n=1)$.

A secondary observer monitored the visual attention of 8 of the infants ( $25 \%$ of the sample), in order to assess interobserver reliability. Length of visual fixation was calculated for each trial for each infant and each observer. A Pearson product-moment correlation was computed between the observations of the primary and the secondary observers and averaged $.97(S D=.06)$.

\section{Results and Discussion}

Figure 1 presents the infants' mean visual fixations as a function of trial type (baseline, posthabituation, and test) for the bimodal audiovisual and the unimodal visual conditions. The primary dependent variable used as the index of discrimination was visual recovery to a change in tempo. Visual recovery is a difference score computed for each infant by subtracting the mean number of seconds the infant spent looking during the two no-change posthabituation trials from the mean number of seconds spent looking during the two test trials. Figure 2 depicts the mean visual recovery scores for the infants in the bimodal audiovisual and the unimodal visual conditions of the present experiment, along with those in Bahrick et al. (2002).

To examine whether 5-month-olds could discriminate a change in tempo under the bimodal audiovisual and the unimodal visual conditions, we conducted a single-sample $t$ test on the infants' mean visual recovery score against the chance value of zero for the bimodal and the unimodal conditions separately (see Figure 2). The results of both tests were significant [bimodal $t(15)=4.27, p=.001$; unimodal $t(15)=3.04, p=.008$, all tests are twotailed]. They indicate that 5-month-old infants were able to discriminate between the two tempos under both the bimodal (audiovisual) and the unimodal (visual) habituation and testing. Furthermore, a two-sample $t$ test comparing the performances of the infants in the unimodal and the bimodal conditions demonstrated no significant difference between them $[t(30)=0.55, p>.1]$. Thus, the infants who received nonredundant unimodal visual events preformed on par with those who received redundant (synchronized auditory and visual) events during habituation and test. Both groups showed robust visual recovery to the change in tempo. These findings contrast with those of the younger, 3-month-old infants in our prior study (Bahrick et al., 2002). The infants in the bimodal condition of that study showed significant visual recovery to the change in tempo $[t(15)=2.30, p=.036]$, whereas those in the unimodal condition did not $[t(15)=1.1, p>.1]$.

We also examined the data at the individual subject level to ascertain whether the significant visual recovery was a result of the group as a whole or was carried primarily by a few infants who had particularly high visual recovery scores. Recovery scores were classified as positive or negative, and a binomial test was conducted to assess whether the number of infants who showed positive visual recovery scores was significantly greater than chance (.50). All 16 of the infants in the bimodal audiovisual condition $(p<.0001)$ and 13 of the 16 infants in the unimodal visual condition $(p<.01)$ showed positive 


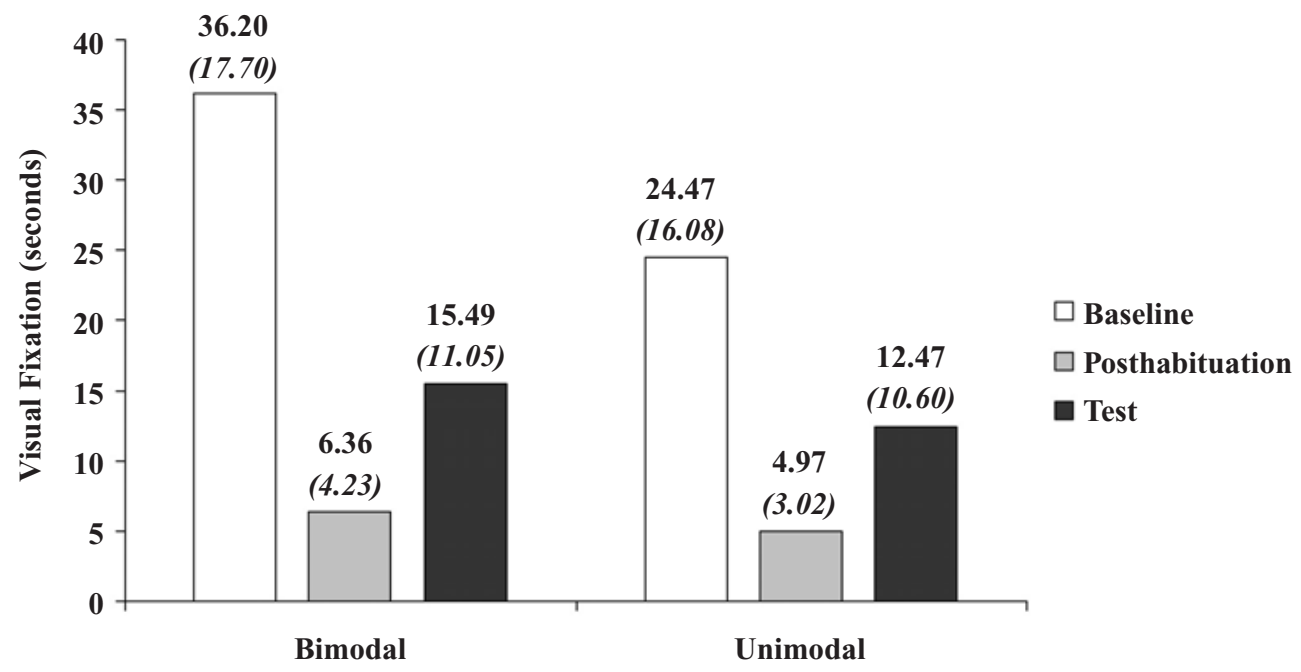

Figure 1. Experiment 1: mean visual fixation scores (and standard deviations) as a function of trial type (baseline, posthabituation, and test) during bimodal redundant audiovisual versus unimodal visual presentations of tempo. Baseline is the mean visual fixation during the first two habituation trials, reflecting initial interest level. Posthabituation is the mean visual fixation during the two no-change trials just after the habituation criterion was met, reflecting final interest level in the habituation event. Test is the mean visual fixation during the two tempo change trials.

recovery scores. Thus, discrimination of tempo following both unimodal visual and bimodal audiovisual exposure was robust and characterized the group as a whole. In contrast, in our prior study with 3-month-olds (Bahrick et al., 2002), a significant number of the infants in the bimodal condition $(13$ of $16 ; p<.01$ ) showed positive visual recovery scores to the change in tempo, but only 9 of the 16 infants in the unimodal condition showed positive visual recovery scores ( $p>.1$; a chance effect). Collapsing across conditions, the performances of the 3month-old and the 5-month-old infants differed significantly, with a greater number of older infants showing positive visual recovery scores $\left[\chi^{2}(1)=12.8, p=\right.$ $.0001]$.

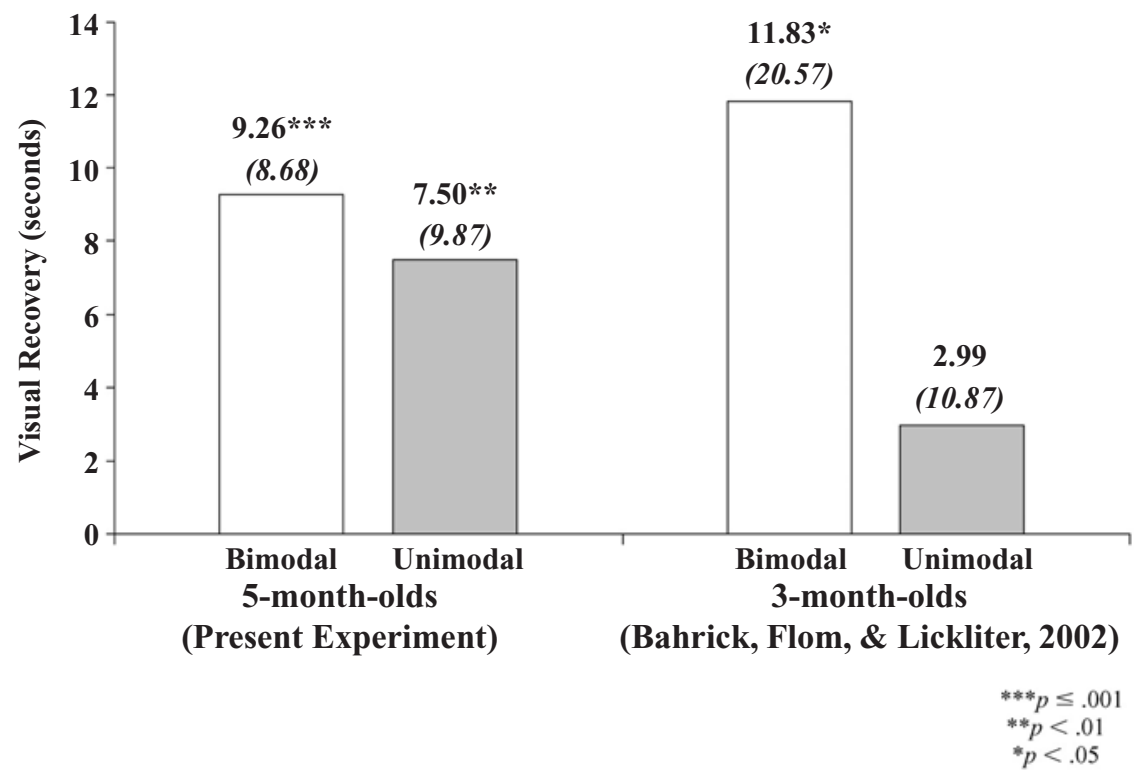

Figure 2. Mean visual recovery scores (and standard deviations) to a change in tempo following redundant bimodal stimulation versus unimodal visual stimulation for the infants in Experiment 1 and those in our prior study (Bahrick, Flom, \& Lickliter, 2002). Visual recovery is a difference score between visual fixation during the test trials and visual fixation during the posthabituation trials. 
Analyses were also performed to assess whether the infants in the present experiment showed any stimulus preferences. Two-way analyses of variance (ANOVAs) were performed on the visual recovery scores, with stimulus tempo (fast vs. slow) and stimulus rhythm (Rhythm 1 vs. 2 ) as main factors for the bimodal and the unimodal conditions separately. The results indicated no main effects or interactions for the infants in the bimodal condition (all $p \mathrm{~s}>.1$ ). Thus, visual recovery did not differ as a function of which tempo or rhythm the infants received for habituation. The results of the unimodal condition indicated a significant main effect of tempo $[F(1,12)=17.2, p=$ $.001]$ and no other significant effects $(p s>.05)$. Apparently, visual recovery was greater for the infants who received the fast tempo for habituation and the slow one for test than for those who received the slow tempo for habituation and the fast one for test. Two-way ANOVAs with stimulus rhythm and tempo as main factors were also performed on processing time as the dependent variable, to determine whether the infants had spent longer, overall, looking at the events as a function of which rhythm or tempo they had received for habituation. No significant main effects or interactions in the unimodal condition were found (all $p \mathrm{~s}>.05$ ), indicating no overall looking preference for one rhythm or tempo over the other. There was, however, a significant main effect of rhythm for the bimodal condition $[F(1,12)=5.46, p=.04]$, indicating that the infants spent more overall time attending to one of the two rhythms.

Secondary analyses were also conducted comparing performance across the bimodal and the unimodal conditions, to determine whether initial interest level (baseline), final interest level (posthabituation; see Figure 1), number of trials to habituation $(M=6.9, S D=2.1$, vs. $M=9.2, S D=4.9$, respectively) or total processing time for the events $(M=137.2, S D=56.7$ vs. $M=125.7$, $S D=60.0$, respectively), differed as a function of condition. Four one-way ANOVAs were conducted, one for each variable, with condition (bimodal or unimodal) as the main factor. Despite the fact that the mean looking times were consistently higher for the bimodal than for the unimodal condition (see Figure 1), the analyses revealed only marginally significant effects for two of the measures [baseline $F(1,30)=3.84, p=.06$, number of trials, $F(1,30)=3.06, p=.09]$ ]. The other two measures did not approach significance $(p s>.1)$. Thus, the infants in the two groups differed marginally in their initial interest and number of trials to habituation and did not differ in their final interest or total processing time for the bimodal versus the unimodal events.

Overall, these results indicate that 5-month-old infants can discriminate between the tempos of two events when they are presented bimodally (audiovisually) and unimodally (visually). These findings contrast with those in our prior study that used the same procedure with 3month-old infants (see Figure 2). Three month-olds detected the change in tempo following bimodal (audio- visual), but not unimodal (visual), presentations of the events. It thus appears that between the ages of 3 and 5 months, infant attention becomes more flexible and detection of tempo is extended from bimodal to unimodal stimulation. These findings support the developmental prediction of the IRH. That is, the facilitating effects of intersensory redundancy for the detection of amodal properties of events (e.g., tempo) appear most pronounced in early development, when the stimulation is novel or difficult, and are attenuated later in development, as the stimulation becomes more familiar through experience with similar events.

If the developmental prediction of the IRH reflects a general developmental principle, the developmental shift from bimodal only to bimodal and unimodal detection of amodal stimulation should be evident in infants' detection of other amodal properties. Since our prior research (Bahrick \& Lickliter, 2000) had also established that 5month-olds could detect a change in the rhythm of these events following bimodal, but not unimodal, stimulation, in Experiment 2 we investigated the development of older infants' detection of rhythm. Eight-month-old infants were chosen for this experiment, since it was thought that our rhythm discrimination task was more difficult than our tempo discrimination task and this would allow the 5-month-olds 3 months' additional experience with natural events.

\section{EXPERIMENT 2 \\ Discrimination of Rhythm in Redundant Bimodal Stimulation Versus Unimodal Visual Stimulation}

In this experiment, we investigated the ability of 8month-old infants to perceive a change in the amodal property of rhythm in the context of redundant bimodal (audiovisual) stimulation versus nonredundant unimodal (visual) stimulation. We used procedures identical to those in Experiment 1, except that we assessed change in rhythm just as we had in our prior study with 5-montholds (Bahrick \& Lickliter, 2000).

\section{Method}

Participants. Forty 8-month-old infants participated. As in the prior study, the infants had a gestation period of at least 38 weeks, they were primarily from middle class homes, and their parents had at least a high school education. There were 21 males and 19 females, and they had a mean age of 243.6 days $(S D=8.1)$. Five of the infants were Caucasian, 33 were of Hispanic origin, 1 was African American, and 1 was Asian. Twenty-four additional infants participated $(38 \%)$, but their data were excluded from the analyses due to fussiness $(n=5)$, falling asleep $(n=2)$, failure to habituate within 20 trials $(n=1)$, and failure to pass the fatigue $(n=12)$ and habituation $(n=4)$ criteria (see the Procedures section for details). Many of the 8-month-old infants appeared to have a difficult time maintaining interest in the procedure and the events, particularly the bimodal events. Nine of the 12 infants whose data were rejected for fatigue/lack of attentiveness were from the bimodal condition. We think that this was likely due to the fact that the task was easy for the 8-month-olds (in- 
fants are successful at discriminating rhythms in the bimodal condition at the younger age of 5 months) and they processed the information quickly and then became bored. In addition, 12 infants (6 from each condition) were tested, but data were not obtained due to equipment failure or experimenter errors, since this was a time at which our lab was integrating new computer equipment and programs that initially did not run reliably.

Stimulus events and Apparatus. The apparatus and stimulus events were identical to those in Experiment 1 and those in Bahrick and Lickliter (2000).

Procedure. The procedure paralleled that in Experiment 1 and Bahrick et al. (2002), except that a change in rhythm, rather than in the tempo of the events, was presented during the test trials. The procedure departed from that in Bahrick and Lickliter (2000) in one respect. In the bimodal condition of Bahrick and Lickliter's (2000) study, the infants received bimodal habituation and unimodal visual test trials depicting a change in rhythm. This necessitated comparing the infants' performance in the test trials with that of a control group that received bimodal habituation and unimodal visual test trials depicting no change in rhythm. The present experiment used the more streamlined procedure of Experiment 1 in which bimodal habituation and bimodal test trials were presented, requiring no control group and creating uniformity across the designs of the two experiments.

The infants were randomly assigned to the bimodal audiovisual $(n=20)$ or the unimodal visual $(n=20)$ condition. In the bimodal condition, the infants received audiovisual habituation to the hammer tapping out one of two distinctive rhythms at one of two tempos. Test trials depicted audiovisual presentations of the hammer tapping a novel rhythm at the familiar tempo. In the unimodal condition, the infants received visual habituation to the hammer tapping out one of the two rhythms at one of the two tempos. Test trials depicted visual presentations of the hammer tapping the novel rhythm at the familiar tempo. As before, rhythm and tempo were counterbalanced across infants, so that half the infants in each condition received one of the tempos for habituation and, within each of these groups, half received one of the rhythms for habituation and the other rhythm for test.
Habituation criteria and procedures were identical to those in Experiment 1. The criteria for identifying fatigue and failure to habituate were also similar, except that the fatigue criterion was somewhat more strict for older infants than for younger infants, since older infants process information more quickly and have greater attentional mobility and their data are typically less variable than those of younger infants. As before, to pass the fatigue criterion, the infants were required to show that they were capable of visual recovery to the toy turtle (a completely different event). Their visual fixation on the final control trial (turtle) was required to be $150 \%$ (rather than $100 \%$ ) of their habituated fixation level (i.e., posthabituation fixation) to the hammer events. The data of 12 infants ( 9 from the bimodal and 3 from the unimodal conditions) were rejected for failing this criterion. These infants, for the most part, appeared to be particularly uninterested in the videos toward the end of the procedure. The remaining infants showed a substantial visual recovery on the final control trial, relative to their habituated (posthabituation) looking level $(M=678 \%, S D=501 \%)$. Also, as before, the fixation level on the final control trial was required to be at least $35 \%$ of that on the initial control trial. The data of 1 infant were rejected for failure to meet this criterion. The remaining infants showed robust visual fixation on the final control trial, relative to that on the initial control trial $(M=187 \%, S D=146 \%)$. In addition, as before, to evaluate whether the infants had, in fact, habituated, we required their mean posthabituation fixation level to the hammer events to be less than their own initial mean fixation level to the hammer events (baseline). The data of 4 infants were rejected ( 2 from each condition) for failure to meet this criterion.

A secondary observer monitored the visual fixations of 15 of the infants $(38 \%)$ across the two conditions. Interobserver reliability was calculated as in Experiment 1 and averaged .96. $(S D=.05)$.

\section{Results and Discussion}

Figure 3 depicts mean visual fixation as a function of trial type (baseline, posthabituation, and test) for the infants in the bimodal (audiovisual) and the unimodal (visual) conditions. Visual recovery to the change in rhythm

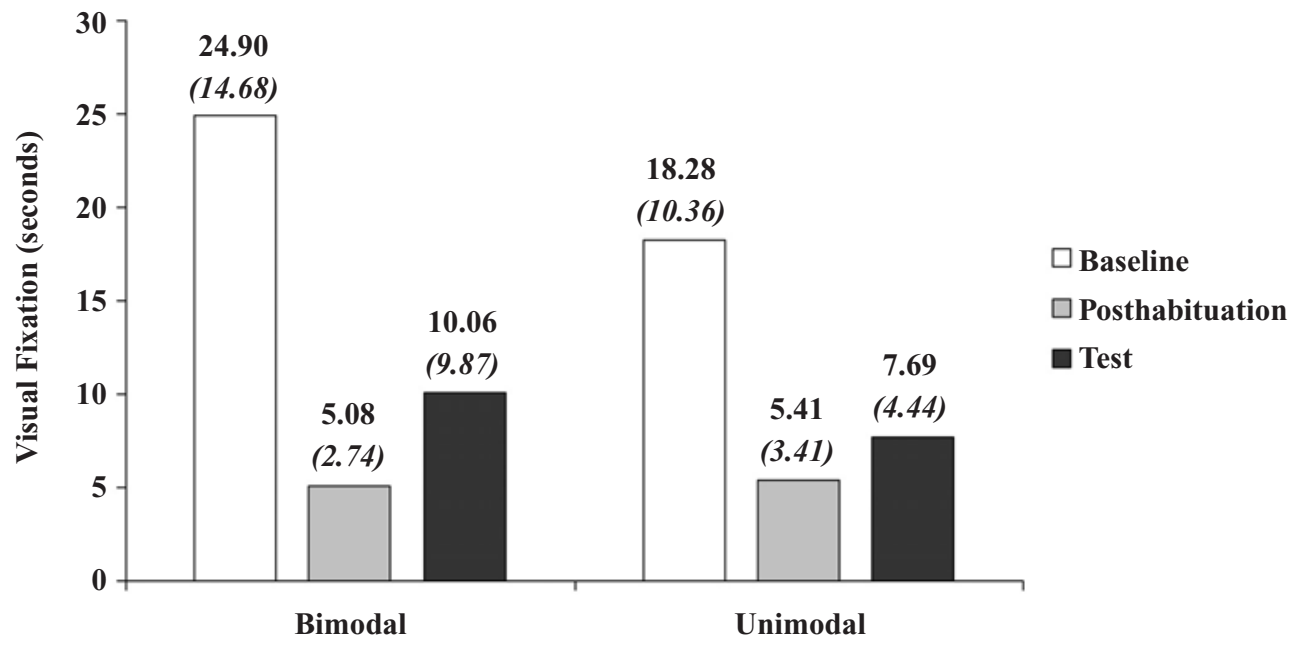

Figure 3. Experiment 2: mean visual fixation scores (and standard deviations) as a function of trial type (baseline, posthabituation, and test) during bimodal redundant audiovisual versus unimodal visual presentations of rhythm. Baseline is the mean visual fixation during the first two habituation trials, reflecting initial interest level. Posthabituation is the mean visual fixation during the two no-change trials just after the habituation criterion was met, reflecting final interest level in the habituation event. Test is the mean visual fixation during the two rhythm change trials. 


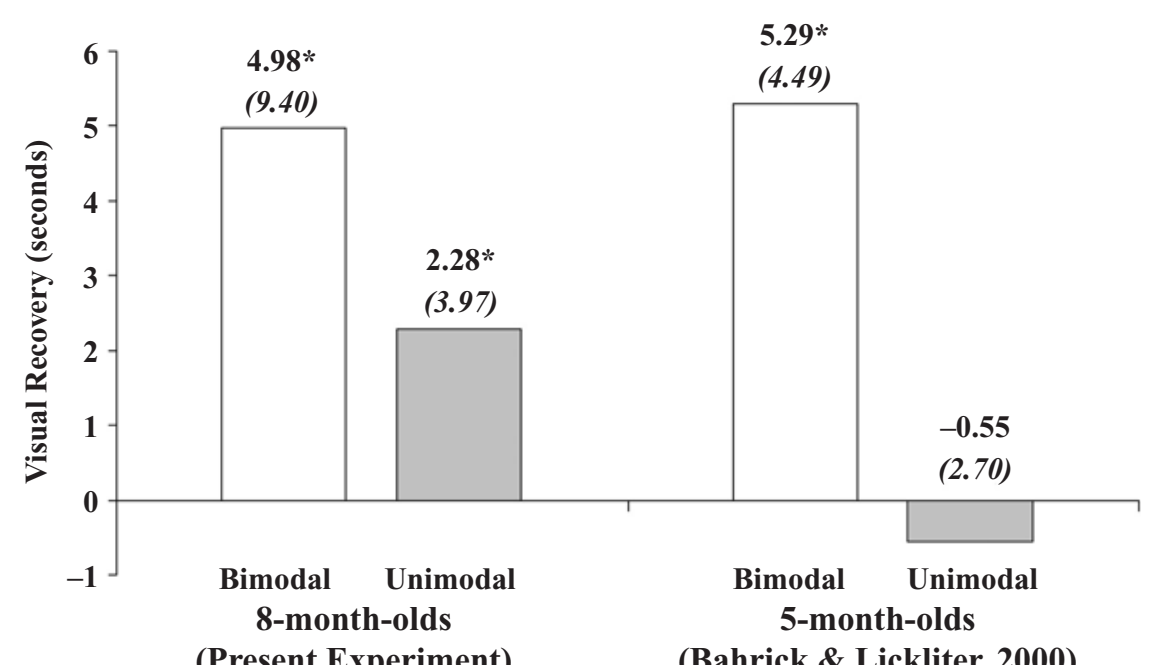

(Present Experiment)

(Bahrick \& Lickliter, 2000)

$$
\begin{aligned}
* * * p & \leq .001 \\
* p & <.05
\end{aligned}
$$

Figure 4. Mean visual recovery scores (and standard deviations) to a change in rhythm following redundant bimodal stimulation versus unimodal visual stimulation for the infants in Experiment 2 and those in our prior study (Bahrick \& Lickliter, 2000). Visual recovery is a difference score between visual fixation during the test trials and visual fixation during the posthabituation trials.

for the infants in each condition was calculated as before and is depicted in Figure 4, along with that from our prior study with 5-month-old infants (Bahrick \& Lickliter, 2000).

To address the main research question of whether 8month-olds can detect a change in rhythm in bimodal audiovisual and in unimodal visual stimulations, singlesample $t$ tests were conducted on the visual recovery scores for each condition. The results indicated a significant visual recovery in both the bimodal $[t(19)=2.37$, $p=.03]$ and the unimodal $[t(19)=2.57, p=.02]$ conditions. In addition, a two-sample $t$ test comparing the performance of infants in the bimodal and the unimodal conditions indicated no significant difference between them $[t(38)=1.18, p>.1]$. Thus, 8 -month-olds showed evidence of detecting a change in the amodal property of rhythm under both bimodal audiovisual and unimodal visual conditions, and their performances did not differ from one another. In contrast, 5-month-olds in the prior study (Bahrick \& Lickliter, 2000) showed significant visual recovery under the bimodal condition $[t(7)=3.37$, $p=.02]$, but not under the unimodal condition $[t(7)=$ $-.58, p>.1]$, and their performances differed significantly from one another.

The data were also examined at the individual subject level as before, to assess whether evidence of rhythm discrimination was consistent across subjects or was carried primarily by a few infants. The results indicated that 16 of the 20 infants in the bimodal condition and 17 of the 20 infants in the unimodal condition showed positive visual recovery scores, results significantly above chance $(p=$
.005 , and .001 , respectively). These findings demonstrate clear evidence of discriminating a change in the amodal property of rhythm at the individual subject level for the infants who received both unimodal visual and bimodal audiovisual events. In contrast, the younger infants in the prior study (Bahrick \& Lickliter, 2000) all showed positive visual recovery scores in the bimodal condition (8 of 8 ; $p=.004)$, whereas only half showed positive visual recovery scores in the unimodal condition (4 of $8 ; p>.1$ ), indicating a chance effect. Collapsing across conditions, the performance of the 5-month-old and 8-month-old infants differed significantly, with a greater number of older infants showing positive visual recovery scores $\left[\chi^{2}(1)=\right.$ $10.4, p=.001]$.

Analyses were also conducted to assess whether the infants showed any preferences for one rhythm over the other or for one tempo over the other. Two-way ANOVAs were performed on the visual recovery scores, with tempo (fast vs. slow) and rhythm (Rhythm 1 vs. 2) as main factors for the bimodal and the unimodal conditions separately. The results indicated no main effects or interactions for infants in either the bimodal or the unimodal condition (all $p \mathrm{~s}>.1$ ). Thus, visual recovery did not differ as a function of which tempo or rhythm the infants received for habituation. In addition, two-way ANOVAs with stimulus rhythm and tempo as main factors were also performed as before on processing time as the dependent variable, to determine whether the infants spent longer, overall, looking at the events as a function of which rhythm or tempo they had received for habituation. The results indicated no significant main effects or interactions in either the bimodal or 
the unimodal condition (all $p \mathrm{~s}>.1$ ). Thus, the infants showed no evidence of any stimulus preferences according to their visual recovery scores or their overall looking time preferences.

Secondary analyses were also conducted, as in Experiment 1 , comparing performance across the bimodal and the unimodal conditions to determine whether initial interest level (baseline), final interest level (posthabituation; see Figure 3), number of trials to habituation $(M=$ $7.0, S D=2.4$, vs. $M=8.0, S D=3.5$, respectively), or total processing time for the events $(M=118.3, S D=$ 74.3 vs. $M=96.8, S D=38.1$, respectively) differed as a function of condition. Four one-way ANOVAs were conducted, one for each variable, with condition (bimodal or unimodal) as the main factor. Despite the fact that the mean looking times were higher for the bimodal than for the unimodal condition (see Figure 3), the analyses revealed no significant effects for any of the measures (all $p \mathrm{~s}>.1$ ). Thus, the infants in the two groups did not differ in their initial interest, final interest, pattern of habituation, or total processing time for the bimodal versus the unimodal events.

Overall, these results demonstrate that 8-months-olds discriminate between two rhythms when they are presented either bimodally (audiovisually) or unimodally (visually). These findings are in contrast with those in our prior study with younger infants (Bahrick \& Lickliter, 2000), where discrimination of bimodal, but not unimodal, visual rhythm was found (see Figure 4). Similar to the findings in Experiment 1 with perception of tempo, perception of rhythm also appears to become more flexible with experience, extending from bimodal contexts in early development to unimodal contexts in later development.

\section{GENERAL DISCUSSION}

In the present study, we assessed the ability of infants to detect a change in the amodal properties of rhythm and tempo in naturalistic events consisting of an object striking a surface. We evaluated the developmental prediction of the IRH (see Bahrick \& Lickliter, 2000, 2002). According to this hypothesis, intersensory redundancy (available in bimodal, but not unimodal, stimulation) is highly salient and directs attention to amodal properties of events in early development. However, later in development, as infants become more experienced perceivers and perceptual differentiation progresses, attention becomes more flexible. Infants then become proficient at detecting amodal properties in the context of unimodal stimulation (where intersensory redundancy is not available), as well as in bimodal stimulation.

The results of Experiment 1 supported our developmental prediction and demonstrated that 5-month-old infants could detect a change in the tempo of a toy hammer tapping (from fast to slow or vice versa) under both bimodal audiovisual and unimodal visual habituation and test conditions. In contrast with our prior results with 3month-olds, using similar procedures (Bahrick et al.,
2002), these more perceptually experienced infants detected the amodal property of tempo even in unimodal stimulation, where no intersensory redundancy specifying tempo was available. Similarly, the results of Experiment 2 also supported our developmental hypothesis and demonstrated that 8-month-old infants could detect a change in the rhythm of the hammer tapping under both bimodal audiovisual and unimodal visual habituation and test conditions. In contrast with our prior results with 5-month-olds (Bahrick \& Lickliter, 2000), these more perceptually experienced infants detected the amodal property of rhythm in unimodal stimulation, where no intersensory redundancy highlighting rhythm was available. These results converge to demonstrate that in later development, with a few months additional experience, attention becomes more flexible and amodal stimulus properties can be detected without the benefit of intersensory redundancy.

This developmental progression is also supported by other recent findings in the developmental literature. For example, infant perception of emotional expressions appears to emerge, first, on the basis of multimodal information and, later in development, on the basis of auditory or visual information (Allen, Dodd, Flom, \& Bahrick, 2003; Walker-Andrews, 1997). Furthermore, infants detect the temporal order of event sequences on the basis of audiovisual information at 4 months, but not on the basis of auditory or visual information alone. By 8 months, infants detect temporal order on the basis of either visual or auditory information alone (Lewkowicz, 2004). These findings reveal a pattern of increasing specificity in perceptual development, from initial detection of amodal information in bimodal sensory stimulation (intermodal) to later detection of amodal information in unimodal stimulation (intramodal).

Taken together with the results of our prior research (Bahrick et al., 2002; Bahrick \& Lickliter, 2000), the present results demonstrate that there is a developmental shift in infants' sensitivity to the amodal properties of events. In early development, when the information to be detected is relatively novel and/or difficult for infants to perceive, the intersensory redundancy available in multimodal stimulation selectively focuses attention on the amodal properties of events (Bahrick et al., 2002; Bahrick \& Lickliter, 2000; Lickliter, Bahrick, \& Honeycutt, 2002, 2004). This facilitates the perception of congruent auditoryvisual patterns of stimulation at a time when infants are not yet particularly experienced at differentiating unitary events from the flux of multimodal stimulation (see Bahrick, 2004; Bahrick \& Lickliter, 2002; Bahrick et al., 2004a). At this phase of development, unimodal stimulation (which provides no intersensory redundancy) typically promotes attention to modality-specific properties (Bahrick et al., 2004b). The present research demonstrates that as infants become more experienced perceivers, attention becomes more flexible, and amodal properties can be detected in unimodal stimulation without the benefit of intersensory redundancy. One possible basis for the developmental shift in attentional flexibility is the 
education of attention (Gibson, 1969) from sensitivity to amodal information in bimodal stimulation to sensitivity to the same amodal information in unimodal stimulation. Investigations of the nature of the educating of attention and its influence on the observed developmental shift are currently underway (Lickliter, Bahrick, \& Markham, 2004).

The present results, however, do not necessarily imply that once infants can detect amodal properties in unimodal stimulation, their sensitivity to these properties is as robust or proficient as their sensitivity to these properties in bimodal stimulation. It may be that greater perceptual experience enables perceivers to perform well under both bimodal and unimodal conditions (i.e., performance is at ceiling). As is likely the case with adult perceivers, if one were to make the task more difficult (e.g., more difficult tempo or rhythm contrasts), a selective advantage for detecting these amodal properties would again become evident in the context of bimodal redundant stimulation. This possibility remains to be tested.

Relatedly, evidence from our studies showing earlier discrimination of changes in tempo than of changes in rhythm should not necessarily be taken as evidence that the perception of tempo is easier or develops prior to the detection of rhythm information (but see Lewkowicz, 2000). The relative difficulty and the order of emergence of these tasks depends, to a large extent, on the nature of the specific stimuli chosen and the difficulty of the contrasts used. Convergent data across a variety of studies, procedures, and stimuli can more adequately address this issue. For this reason, our research has focused on making direct comparisons between detection of a given property (e.g., tempo) under different conditions (e.g., unimodal vs. bimodal), contexts, and ages. This allows for conclusions that are more easily generalized to the natural environment.

Infant sensitivity to intersensory redundancy helps to explain how, in a predominately multimodal environment, perceptual learning can initially be guided and constrained by the detection of amodal relations uniting patterns of stimulation from unitary events and then, later, be extended to the detection of amodal properties in unimodal contexts. The environment provides a diverse array of multimodal and unimodal stimulation, and which properties of events will be perceived, learned, and remembered at different times and different points in development is determined, in large part, by selective attention. Knowledge of what guides selective attention and how it changes developmentally is essential for successful theories of perception, learning, and memory.

\section{REFERENCES}

Allen, M., Dodd, K., Flom, R., \& Bahrick, L. E. (2003, April). Intersensory redundancy: Five- and seven-month-olds' perception of affect. Paper presented at the Society for Research in Child Development, Tampa, FL.

BAHRICK, L. E. (1983). Infants' perception of substance and temporal synchrony in multimodal events. Infant Behavior \& Development, $\mathbf{6}$, 429-451.
BAHRICK, L. E. (1987). Infants' intermodal perception of two levels of temporal structure in natural events. Infant Behavior \& Development, 10, 387-416.

BAHRICK, L. E. (1988). Intermodal learning in infancy: Learning on the basis of two kinds of invariant relations in audible and visible events. Child Development, 59, 197-209.

BAHRICK, L. E. (1992). Infants' perceptual differentiation of amodal and modality-specific audio-visual relations. Journal of Experimental Child Psychology, 53, 180-199.

BAHRICK, L. E. (1994). The development of infants' sensitivity to arbitrary intermodal relations. Ecological Psychology, 6, 111-123.

BAHRICK, L. E. (2001). Increasing specificity in perceptual development: Infants' detection of nested levels of multimodal stimulation. Journal of Experimental Child Psychology, 79, 253-270.

BAHRICK, L. E. (2004). The development of perception in a multimodal environment. In G. Bremner \& A. Slater (Eds.), Theories of infant development (pp. 90-120). Malden, MA: Blackwell.

BAhrick, L. E., Flom, R., \& LiCKLITER, R. (2002). Intersensory redundancy facilitates discrimination of tempo in 3-month-old infants. $\underline{D e-}$ velopmental Psychobiology, 41, 352- 363.

BAHRICK, L. E., \& LICKLITER, R. (2000). Intersensory redundancy guides attentional selectivity and perceptual learning in infancy. $\underline{D e-}$ velopmental Psychology, 36, 190-201.

BAHRICK, L. E., \& LICKLITER, R. (2002). Intersensory redundancy guides early cognitive and perceptual development. In R. V. Kail (Ed.), $A d-$ vances in child development and behavior (Vol. 30, pp. 153-187). New York: Academic Press.

BAHrick, L. E., Lickliter, R., \& Flom, R. (2004a). Intersensory redundancy guides infants' selective attention, perceptual, and cognitive development. Current Directions in Psychological Science, $\mathbf{1 3}$ 99-102.

BAHRICK, L.E., LiCKLITER, R., \& Flom, R. (2004b). Up versus down: The role of intersensory redundancy in infants' sensitivity to the orientation of moving objects. Manuscript under review.

Bahrick, L. E., Lickliter, R., Shuman, M., Batista, L., \& Grandez, C. (2003, April). Infant discrimination of voices: Predictions from the intersensory redundancy hypothesis. Paper presented at the Society for Research in Child Development, Tampa, FL.

BAhrick, L. E., Moss, L., \& FADIL, C. (1996). The development of visual self-recognition in infancy. Ecological Psychology, 8, 189-208.

BARRERA, M., \& MAURER, D. (1981). Recognition of mother's photographed face by the three month old infant. Child Development, 52, 714-716.

Bertenthal, B. I., Haith, M. M., \& Campos, J. J. (1983). The partiallag design: A method for controlling spontaneous regression in the infant-control habituation paradigm. Infant Behavior \& Development, 6, 331-338.

CAlvert, G. A., SPEnce, C., \& Stein, B. E. (2004). The handbook of multisensory processes. Cambridge, MA: MIT Press.

Caron, A. J., Caron, R. F., Caldwell, R. C., \& Weiss, S. J. (1973). Infant perception of the structural properties of the face. Developmental Psychology, 19, 385-399.

Cohen, L., \& Strauss, M. S. (1979). Concept acquisition in the human infant. Child Development, 50, 419-424.

DeCasper, A. J, \& Fifer, W. (1980). Of human bonding: Newborns prefer their mothers' voices. Science, 208, 1174-1176.

DoDD, B. (1979). Lip reading in infants: Attention to speech presented in-and-out of synchrony. Cognitive Psychology, 11, 478-484.

FAGAN, J. F. (1972). Infants' recognition memory for faces. Journal of Experimental Child Psychology, 14, 453-476.

FAGAN, J. F. (1976). Infants' recognition of invariant features of faces. Child Development, 47, 627-638.

Gibson, E. J. (1969). Principles of perceptual learning and development. New York: Appleton-Century-Crofts.

Gogate, L. J., \& BAHRICK, L. E. (1998). Intersensory redundancy facilitates learning of arbitrary relations between vowel sounds and objects in seven-month-old infants. Journal of Experimental Child Psychology, 69, 1-17.

HERNANDEZ-REIF, M., \& BAHRICK, L. E. (2001). The development of visual-tactual perception of objects: Amodal relations provide the basis for learning arbitrary relations. Infancy, 2, 51-72. 
Horowitz, F. D., Paden, L., Bhana, K., \& Self, P. (1972). An infantcontrol procedure for studying infant visual fixations. Developmental Psychology, 7, 90.

KUHL, P. K., \& MELTZOFF, A. N. (1984). The intermodal representation of speech in infants. Infant Behavior \& Development, 7, 361-381.

LeWKOWICZ, D. J. (1996). Infants' response to the audible and visible properties of the human face: Role of lexical syntactic content, temporal synchrony, gender, and manner of speech. Developmental Psychology, 32, 347-366.

LEWKOWICZ, D. J. (2000). The development of intersensory temporal perception: An epigenetic systems/limitations view. Psychological Bulletin, 126, 281-308.

LEWKOWICZ, D. J. (2004). Perception of serial order in infants. Developmental Science, 7, 175-184.

LICKLITER, R., \& BAHRICK, L. E. (2000). The development of infant intersensory perception: Advantages of a comparative, convergentoperations approach. Psychological Bulletin, 126, 260-280.

LiCKLITER, R., BAHRICK, L. E., \& HONEYCUTT, H. (2002). Intersensory redundancy facilitates perceptual learning in bobwhite quail embryos. Developmental Psychology, 38, 15-23.

LiCKLITER, R., BAHRICK, L. E., \& HoNEYCUTT, H. (2004). Intersensory redundancy enhances memory in bobwhite quail embryos. Infancy, 5, 253-269.

Lickliter, R., BAHrick, L. E., \& MARKham, R. (2004, May). Intersensory redundancy can educate attention during prenatal development. Paper presented at the International Conference on Infant Studies, Chicago.
Miller, C. L. (1983). Developmental changes in male/female voice classification by infants. Infant Behavior \& Development, 6, 313330.

Miller, C. L., Younger, B. A., \& Morse, P. A. (1982). The categorization of male and female voices in infancy. Infant Behavior \& Development, 5, 143-159.

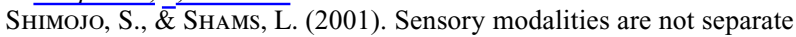 modalities: Plasticity and interactions. Current Opinion in Neurobiology, 11, 505-509.

SlAter, A., QuinN, P. C., Brown, E., \& Hayes, R. (1999). Intermodal perception at birth: Intersensory redundancy guides newborn infants' learning of arbitrary auditory-visual pairings. Developmental Science, 2, 333-338.

SPELKE, E. S. (1979). Perceiving bimodally specified events in infancy. Developmental Psychology, 15, 626-636.

Spelke, E. S., Born, W., \& ChU, F. (1983). Perception of moving, sounding objects by four-month-old infants. Perception, 12, 719-732.

Stein, B. E., \& Meredith, M. A. (1993). The merging of the senses. Cambridge, MA: MIT Press.

WALKER-ANDREWS, A. S. (1997). Infants' perception of expressive behaviors: Differentiation of multimodal information. Psychological Bulletin, 121, 437-456.

(Manuscript received October 14, 2003; revision accepted for publication May 6, 2004.) 